\title{
Incorporation of the Blood Vessel Wall into Electroporation Simulations
}

\author{
L. Silve ${ }^{1}$, R. Qasrawi ${ }^{1}$ and A. Ivorra ${ }^{1,2}$ \\ ${ }^{1}$ Universitat Pompeu Fabra/Department of Information and Communication Technologies, Barcelona, Spain \\ ${ }^{2}$ Serra Húnter Fellow, Universitat Pompeu Fabra, Barcelona, Spain
}

\begin{abstract}
Electroporation can be used in living tissues in order to enhance the penetration of drugs or DNA plasmids or to destroy undesirable cells and it is typically performed by applying pulsed high voltages across needle electrodes. When used for ablation, it is often claimed that, in contrast with thermal ablation techniques, electroporation is not significantly impacted by the presence of large blood vessels because the heat sinking characteristic of these is not relevant for the electric field distribution. However, large blood vessels do distort the electric field distribution because of their high inner conductivity and should be modeled during treatment planning. For such purpose, vessels may be simply modeled as homogeneous regions whose conductivity is equal to that of the blood. Nevertheless, vessels are not just blood filled cavities within parenchyma; blood vessels contain a layered wall. The purpose of the present study is to check whether the blood vessel wall needs to be incorporated into the simulations. For that, a vessel wall electrical model has been implemented and it has been incorporated into $2 \mathrm{D}$ and $3 \mathrm{D}$ simulations in which treatment of a region that comprises a $5 \mathrm{~mm}$ thick artery within liver was modeled. The three main layers of a vessel wall (the intima, the media and the adventitia) were modeled as homogeneous materials whose conductivity depends on the electric field magnitude. The simulations show that the electric field error when the wall model is not incorporated is only marginally significant at the close vicinity of the vessel for low applied fields. Errors are insignificant beyond 1 or $2 \mathrm{~mm}$. We conclude that in most electroporation scenarios it will not be necessary to simulate the blood vessel wall.
\end{abstract}

Keywords - Blood vessel, Blood vessel wall, Simulation, Electric field, Electroporation.

\section{INTRODUCTION}

Electroporation is the phenomenon in which cell membrane permeability is increased by exposing the cell to high intensity electric fields. In living tissues, such permeabilization boost can be used in order to enhance the penetration of drugs or DNA plasmids or to destroy undesirable cells and it is typically performed by applying pulsed high voltages across needle electrodes.

When used for ablation, and particularly in the context of liver tumors, it is often claimed that, in contrast with thermal ablation techniques, such as radiofrequency ablation, electroporation based techniques are unaffected by the presence of large blood vessels in the treated region. The ra- tionale for that claim is that, while thermal methods are negatively impacted by the heat sinking effect of large vessels, electroporation is not impacted because its local efficacy depends on the electric field magnitude and the electric field distribution is almost independent of thermal effects. Blood vessels, however, do distort the electric field distribution because of their relative high inner conductivity [1]. In a past numerical study focused on liver ablation by irreversible electroporation (IRE), we noticed that, although the presence of large blood vessels does not distort very significantly the treated volume, sub-lethal regions (i.e. too low electric field magnitude) can appear around the blood vessels [2]. The presence of such sub-lethal regions has also been reported recently in another numerical study [3]. Since under-treatment may imply that tumor cells remain viable after treatment and that tumors may reappear in months or years, this is an issue that needs to be carefully considered.

In the numerical study reported in [2], the vessels were simply modeled as homogeneous regions with a conductivity equal to that of the blood (this model is referred to as the "blood model" in the following sections). However, blood vessels are not just blood filled cavities within parenchyma; blood vessels contain a wall that separates blood from the parenchyma. In the present study, the blood vessel wall is incorporated into numerical simulations of the electric field distribution during electroporation and the results are compared with simulations using the simple blood model.

\section{Construction of THE MOdel}

\section{A. Geometry, dimensions and composition of the blood vessels wall}

The blood vessel wall basically consists of three layers. From the inside to the outside: the first layer is the intima, which - from an electrical point of view as later justified can be considered to consist in a tight unicellular endothelial layer. The second layer is the media, which mainly consists of concentric layers of Vascular Smooth Muscle Cells (VSMCs). And last, the adventitia is formed by a loose connective tissue.

For physiological reasons, in the human body, blood vessels diameters and wall thicknesses vary a lot. Figure 1 indicates the composition and dimensions of various blood 
vessels. Arteries walls are rich in VSMCs and they are relatively thick in relation to their diameter. On the other hand, vein walls are thinner and poor in VSMCs.
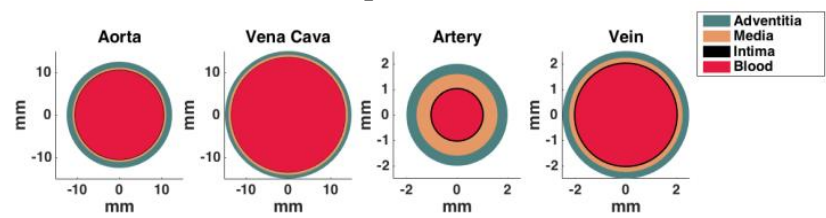

Fig. 1 To scale representation of various blood vessels. From inside to outside, the wall layers are: The intima (black line), the media and the adventitia. Adapted from data in [4].

Because of our interest in IRE ablation of liver tumors, the numerical study implemented here models such clinical scenario. In particular, we have decided to simulate treatment of a region that comprises a branch of the hepatic artery. The branch is assumed circular with an external diameter of $5 \mathrm{~mm}$ and a wall thickness of $1 \mathrm{~mm}$. Vessel dimensions have been taken from [5].

\section{B. Electrical model rationale}

Here it was assumed that the electrical conductivity of solid living tissues strongly depends on the local electric field magnitude. This assumption is done in order to incorporate the effect of the electroporation phenomenon on the conductivity of tissues [6]. In particular, the dependence of the conductivity on the electric field was modeled with a specific sigmoid function for each solid tissue:

$$
\sigma=a_{0}+\frac{a_{1}-a_{0}}{1+e^{-\frac{(E-b)}{c}}}
$$

$\sigma$ : electric field dependant tissue conductivity $[\mathrm{S} / \mathrm{m}]$

$E$ : electric field magnitude $[\mathrm{V} / \mathrm{cm}]$

$a_{0}$ : minimum tissue conductivity (no electroporation)

$a_{1}$ : maximum conductivity (fully electroporation)

$b, c$ : sigmoid shape parameters

Figure 2 a displays the sigmoid function used for the liver tissue. The four values for its parameterization were extracted from [7] for $100 \mu$ s pulses.

No empirical data was found in the literature on the electrical properties of each one of the three layers of the blood vessel wall. Parameterization was therefore performed by doing reasonable assumptions with data from studies on other tissues. The resulting conductivity models are displayed in Figure 2. What follows succinctly summarizes how the models were developed.

The intima layer consists in a unicellular endothelium on a thin elastic membrane made of connective tissue. The thin connective tissue can be considered as negligible from an electrical point of view because of its low resistivity due to the low density of cells. The endothelium, while being thinner than the membrane, imposes a high electrical resistance because the cells are tightly packaged. However, because of its extreme thinness the endothelium is easily electroporated and its resistance dramatically decreases. The implemented conductivity model for the intima is displayed in Figure $2 \mathrm{~d}$. Parameterization was performed by assuming that the endothelium is equivalent to the epithelial layer in the cornea [8].

The media is mainly composed of VSMCs. Therefore it was assumed that its sigmoid function parameters must be similar to the ones of skeletal muscles. Muscle conductivity data was obtained from [9-13]. Muscle tissues are highly anisotropic and those studies indicate different conductivity values for perpendicular and parallel directions to the muscle fibers. Due to the arrangement of VSMCs in blood vessels here conductivity values for perpendicularly oriented fibers were preferred.

The adventitia is a loose connective tissue in which cells, mainly fibroblasts, are quite sparsely distributed in an Extra Cellular Matrix (ECM). This ECM is composed of collagen fibers, elastic fibers and fundamental substance. The fundamental substance is an amorphous and highly hydrated gel. Given its composition, high conductivity - and moderate impact of electroporation on conductivity - can be expected for this layer. Loose connective tissue is present in the dermis. Therefore, here available data from the lower layer of the skin was taken for a rough approximation of the adventitia properties [14, 15]. No distinct data for dermis and epidermis could be found. The conductivity values may be underestimated because the epidermis is composed of epithelial cells and keratin that are less conductive than the connective tissue of the dermis. Conductivity data for the epineurium - which is the wall of lose connective tissue that surrounds the nerves - was also taken into account for the model [16].
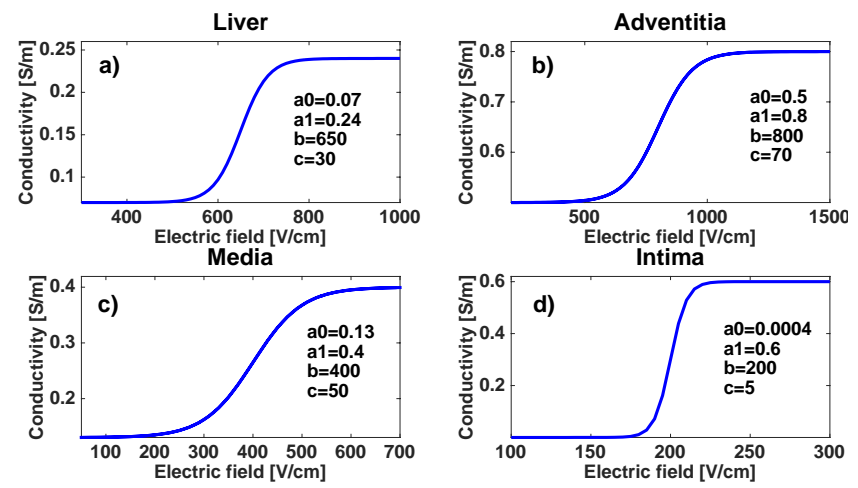

Fig. 2: Conductivity models used in this study.

Lack of empirical data does not allow us to be completely sure about the accuracy of the conductivity models displayed in Figure 2. However, it must be noted that we have checked (not reported here) that by amply varying various parameters of the models, such as the conductivity of the adventitia $(\mathrm{a} 0=0.3 \mathrm{a} 1=0.6)$ or the electroporation 
threshold of the media $(b=500)$, the qualitative conclusions of this study do not change.

Last, the conductivity of blood is set at $0.6 \mathrm{~S} / \mathrm{m}$ as mean value of what can be encountered in the literature $[2,12]$.

\section{Computer simulations}

Computer simulations of the electric field distribution that appears in tissues when a high voltage is applied across two electrodes were performed with a software platform (COMSOL Multiphysics 4.4) based on the finite element method.

A first study was carried out in 2D. The simulation model includes a large square of liver tissue, two needles of $1 \mathrm{~mm}$ of diameter, $16 \mathrm{~mm}$ spaced and a model of blood vessel. Details of the geometry are given in Figure 3. A voltage is then imposed between the two electrodes. A second study was carried out in 3D. An overview of the geometry is displayed in Figure 3. In 3D, needles are finite, ( $2 \mathrm{~cm}$ long) and perpendicular with the vessel. The other dimensions are the same as the ones used in the 2D study.

It must be noted that, due to the extreme thinness of the intima, and for computational issues, this layer was not modeled as a region but as an interface between two regions (the blood and the media).
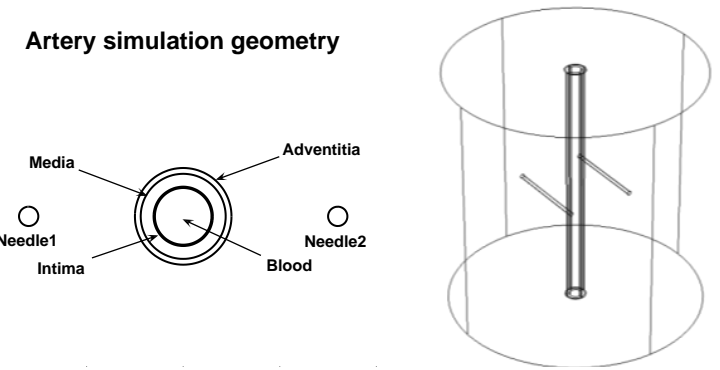

Fig. $32 \mathrm{D}$ artery simulation geometry (left). The needle 1 is at the ground and the needle 2 at a potential P (The vessel and the needles are infinitely parallel). 3D geometry overview (right), needles are $2 \mathrm{~cm}$ long and perpendicular to the vessel.

\section{RESULTS AND DISCUSSION}

\section{A. $2 D$ simulation results}

The simulated electric field distribution is compared in Figure 4 for the simple blood model and the refined model including the wall. It can be observed that differences between the two simulations are subtle and almost only noticeable in the close vicinity of the vessel.

Interestingly, the differences are larger where the sublethal regions appeared around the blood vessels in the IRE study reported in [2]. That is, at the vessel poles perpendicular to the electric field (axis defined by the two electrodes).
By modeling the wall it appears that those regions of depressed electric field magnitude are less intense. However, it must be noted that the electric field magnitude is still significantly lower at those regions and the cautionary remark in [2] still remains in effect. That is, clinicians performing IRE should look for signs of tumor recurrence around the vessels because under-treatment may have occurred there.
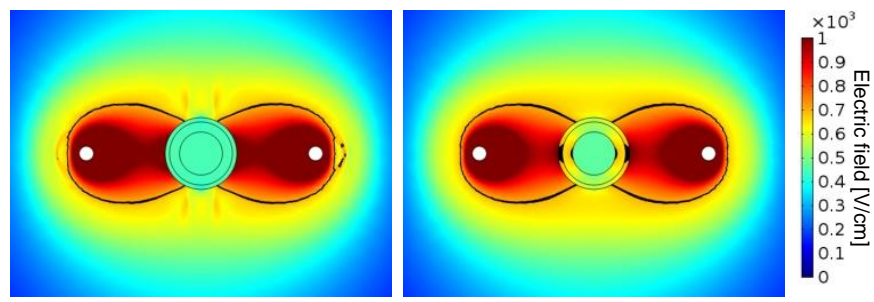

Fig. 4 Simulated electric field distributions for the simple "blood model" (left) and for the model including the wall (right) when $1600 \mathrm{~V}$ are applied across the electrodes. The black line would indicate the boundary IRE treated region $(\mathrm{E}>700 \mathrm{~V} / \mathrm{cm}$, IRE threshold from [7]) for protocol consisting of 80 pulses of $100 \mu \mathrm{s}$.

To quantify the impact of the wall on the liver electroporation, the error between the two models is calculated. The error is defined by the following formula:

$$
\text { error }=\frac{E_{\text {with_wall_model }}-E_{\text {blood_model }}}{E_{\text {blood_model }}} \times 100
$$

The error between the two models around the vessel is displayed in Figure 5.

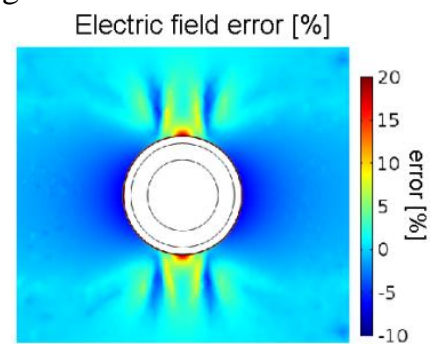

Fig. 5 Electric field magnitude error between simulations using the "blood model" and simulations using the model that includes the wall when $1600 \mathrm{~V}$ are applied across the electrodes. (The vessel is masked for clarity).

It must be noted that the above case is not a benign case in terms of error. The error strongly depends on the magnitude of the applied voltage across the electrodes. This occurs because the impact of the wall depends on its permeabilization status. The dependency of the error on the applied voltage is illustrated in Figure 6 in which the median error in different areas defined around the vessel is obtained.

For applied voltages below $800 \mathrm{~V}$, the wall tissues are not electroporated and they act as a shield against the blood shunting effect. As a consequence, the currents circumvent the vessel and the relative errors can be locally significant 
$(<20 \%)$. On the other hand, for applied voltages above $1600 \mathrm{~V}$ all wall tissues are electroporated and the wall has a conductivity similar to that of blood and both models, with and without wall, produce almost indistinguishable results.
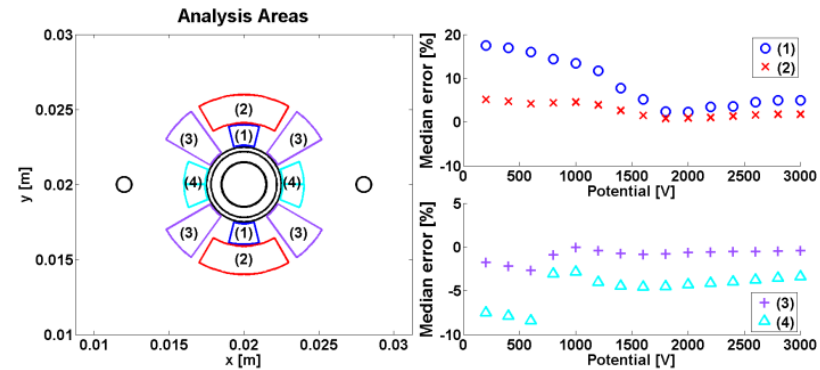

Fig. 6 Median electric field error against the voltage across electrodes for four different areas defined around the vessel. The analysis areas were chosen after the results displayed on Figure 5 in order to highlight the dependency of the results on the location.

\section{B. $3 D$ simulation results}
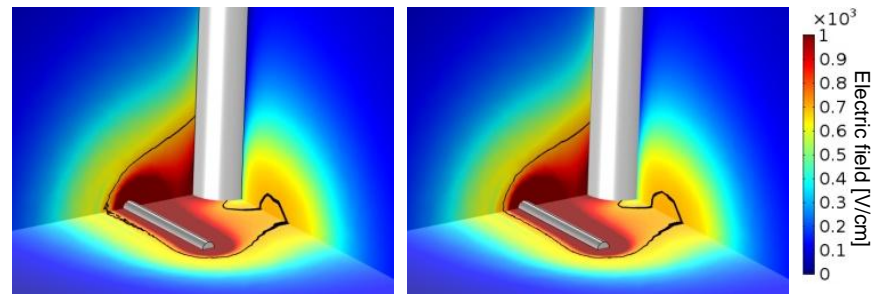

Fig. 7 Simulated electric field distributions for the simple "blood model" (left) and for the model including the wall (right) when $1600 \mathrm{~V}$ are applied across two parallel $2 \mathrm{~cm}$ long electrodes perpendicular to the vessel. The black line represents the boundary of region E $>700 \mathrm{~V} / \mathrm{cm}$ (see Figure 4).

Again it can be observed that differences between the two models can only be (barely) noticed around the vessel.

\section{Conclussions}

The vessel wall does not have a significant impact on the macroscopic electric field distribution and, therefore, it will not have a significant impact on the total treated volume. On the other hand, it may have a significant - but mild (relative impact on $E<20 \%$ ) and punctual - impact on the electric field distribution around the close vicinity of the vessel for relatively low applied fields.

It can be concluded that for most electroporation scenarios, and particularly for IRE, the simple "blood model" will be accurate enough for determining the influence of blood vessels in computer simulations of the electric field.

\section{ACKNOWLEDGMENT}

This work was supported in part by the Spanish Government through grants TEC2010-17285 and TEC2014-52383C3 (TEC2014-52383-C3-2).

\section{CONFLict OF INTEREST}

L. S., R. Q. and A.I. have no conflicts of interest to declare.

\section{REFERENCES}

1. Marčan M, Kos B, Miklavčič D (2015) Effect of Blood Vessel Segmentation on the Outcome of Electroporation-Based Treatments of Liver Tumors. PLoS One 10:e125591.

2. Qasrawi R, Ivorra A (2014) Impact of liver vasculature on electric field distribution during electroporation treatments : an anatomically realistic numerical study. 6th Eur. Conf. Int. Fed. Med. Biol. Eng. pp 573-576

3. Golberg A, Bruinsma BG, Uygun BE, Yarmush ML (2015) Tissue heterogeneity in structure and conductivity contribute to cell survival during irreversible electroporation ablation by "electric field sinks." Sci Rep 5:8485.

4. Burton AC (1954) Relation of structure to function of the tissues of the wall of blood vessels. Physiol Rev 34:619-642.

5. Daghfous A, Baraket O, Bedioui H, et al. (2011) Variations anatomiques de l'artère hépatique et applications pratiques: A propos de 33 cas et revue de la littérature. Tunis Med 89:452-457.

6. Ivorra A (2010) Tissue Electroporation as a Bioelectric Phenomenon: Basic Concepts. In: Rubinsky B (ed) Irreversible Electroporation. Springer Berlin Heidelberg, pp 23-61

7. Miklavcic D, Sel D, Cukjati D, et al. (2004) Sequential finite element model of tissue electropermeabilisation. Conf Proc IEEE Eng Med Biol Soc 5:3551-3554.

8. Guimera A, Ivorra A, Gabriel G, Villa R (2010) Non-invasive assessment of corneal endothelial permeability by means of electrical impedance measurements. Med Eng Phys 32:1107-1115.

9. Martin JB, L. Young J, N. Benoit J, A. Dean D (2000) Gene Transfer To Intact Mesenteric Arteries by Electroporation. J Vasc Res 37:372-378.

10. Dean DA (2005) Nonviral gene transfer to skeletal, smooth, and cardiac muscle in living animals. Am J Physiol Physiol 289:C23345.

11. Miklavcic D, Snoj M, Zupanic A, et al. (2010) Towards treatment planning and treatment of deep-seated solid tumors by electrochemotherapy. Biomed Eng Online 9:1-12.

12. Miklavčič D, Pavšelj N (2006) Electric properties of tissues. Wiley Encycl Biomed Eng 209:1-12. doi: 10.1.1.65.7364

13. Čorović S, Mir LM, Miklavčič D (2012) In vivo muscle electroporation threshold determination: Realistic numerical models and in vivo experiments. J Membr Biol 245:509-520.

14. Corovic S, Lackovic I, Sustaric P, et al. (2013) Modeling of electric field distribution in tissues during electroporation. Biomed Eng Online 12:16.

15. Pavšelj N, Miklavčič D (2011) Resistive heating and electropermeabilization of skin tissue during in vivo electroporation: A coupled nonlinear finite element model. Int J Heat Mass Transf 54:2294-2302.

16. Mortimep JT, Western C (1991) a Numerical Analysis of the Electric Field. 13:912-913.

Author: Antoni Ivorra

Institute: Universitat Pompeu Fabra

Street: Roc Boronat 138

City: Barcelona E-081018

Country: Spain

Email: antoni.ivorra@upf.edu 
